\title{
Human Touch Receptors Are Sensitive to Spatial Details on the Scale of Single Fingerprint Ridges
}

\author{
Ewa Jarocka, ${ }^{1}{ }^{\circledR}$ J. Andrew Pruszynski, ${ }^{1,2,3,4,5}$ and Roland S. Johansson ${ }^{1}$ \\ ${ }^{1}$ Physiology Section, Department of Integrative Medical Biology, Umeå University, Umeå 901 87, Sweden, ${ }^{2}$ Department of Physiology and \\ Pharmacology, Western University, London, Ontario N6A 5C1, Canada, ${ }^{3}$ Department of Psychology, Western University, London, Ontario N6A \\ $5 \mathrm{C} 2$, Canada, ${ }^{4}$ Robarts Research Institute, Western University, London, Ontario N6A 5B7, Canada, and ${ }^{5}$ Brain and Mind Institute, Western \\ University, London, Ontario N6A 3K7, Canada
}

Fast-adapting type 1 (FA-1) and slowly-adapting type 1 (SA-1) first-order tactile neurons provide detailed spatiotemporal tactile information when we touch objects with fingertips. The distal axon of these neuron types branches in the skin and innervates many receptor organs associated with fingerprint ridges (Meissner corpuscles and Merkel cell neurite complexes, respectively), resulting in heterogeneous receptive fields whose sensitivity topography includes many highly sensitive zones or "subfields." In experiments on humans of both sexes, using raised dots that tangentially scanned the receptive field we examined the spatial acuity of the subfields of FA-1 and SA-1 neurons and its constancy across scanning speed and direction. We report that the sensitivity of the subfield arrangement for both neuron types on average corresponds to a spatial period of $\sim 0.4 \mathrm{~mm}$ and provide evidence that a subfield's spatial selectivity arises because its associated receptor organ measures mechanical events limited to a single papillary ridge. Accordingly, the sensitivity topography of a neuron's receptive fields is quite stable over repeated mappings and over scanning speeds representative of real-world hand use. The sensitivity topography is substantially conserved also for different scanning directions, but the subfields can be relatively displaced by directiondependent shear deformations of the skin surface.

Key words: hand; human; microneurography; papillary ridges; receptive field; tactile neurons

Significance Statement

The branching of the distal axon of human first-order tactile neurons with receptor organs associated with fingerprint ridges (Meissner and Merkel end-organs) results in cutaneous receptive fields composed of several distinct subfields spread across multiple ridges. We show that the subfields' spatial selectivity typically corresponds to the dimension of the ridges $(\sim 0.4$ $\mathrm{mm}$ ) and a neuron's subfield layout is well preserved across tangential movement speeds and directions representative of natural use of the fingertips. We submit that the receptor organs underlying subfields essentially measure mechanical events at individual ridges. That neurons receive convergent input from multiple subfields does not preclude the possibility that spatial details can be resolved on the scale of single fingerprint ridges by a population code.

\section{Introduction}

The distal axon of most types of first-order tactile neurons branches in the skin such that a neuron innervates many spatially segregated receptor organs (Cauna, 1959; Lindblom and Tapper, 1966; Brown and Iggo, 1967; Goldfinger, 1990; Vallbo et al., 1995; Paré et al., 2002; Nolano et al., 2003; Wessberg et al., 2003;

\footnotetext{
Received July 3, 2020; revised Feb. 4, 2021; accepted Feb. 4, 2021.

Author contributions: E.J., J.A.P., and R.S.J. designed research; E.J. and R.S.J. performed research; E.J. and R.S.J. analyzed data; E.J., J.A.P., and R.S.J. wrote the paper.

This work was supported by Swedish Research Council Projects 22209 and 01635 and by a Long-Term Fellowship from the Human Frontier Science Program (J.A.P). We thank Carola Hjältén, Göran Westling, Anders Bäckström, and Per Utsi for technical support.

The authors declare no competing financial interests.

Correspondence should be addressed to Ewa Jarocka at ewa.jarocka@umu.se.

https://doi.org/10.1523/JNEUROSCI.1716-20.2021

Copyright $\odot 2021$ the authors
}

Provitera et al., 2007; Lesniak et al., 2014; Kuehn et al., 2019; Neubarth et al., 2020). For the glabrous skin of the human hand, this applies to the fast-adapting type 1 (FA-1) and the slowlyadapting type 1 (SA-1) neurons which innervate Meissner corpuscles and Merkel cell neurite complexes, respectively, and account for the fingertips' exquisite tactile spatial acuity (Vallbo and Johansson, 1984). The branching results in heterogeneous receptive fields with multiple highly sensitive zones (hereinafter also referred to as subfields) seemingly randomly distributed within a circular or elliptical area typically covering five to ten fingerprint ridges (Johansson, 1978; Phillips et al., 1992; Pruszynski and Johansson, 2014).

We have suggested that the subfield arrangement of FA-1 and SA-1 neurons is a functional determinant of the fingertips' high tactile spatial resolution (Pruszynski and Johansson, 2014; Pruszynski et al., 2018). Briefly, the spacing between neurons' 


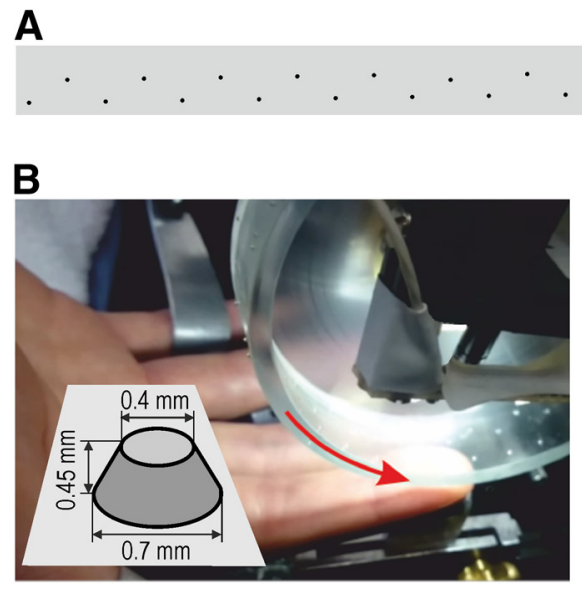

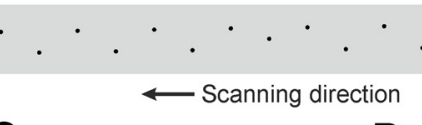

C

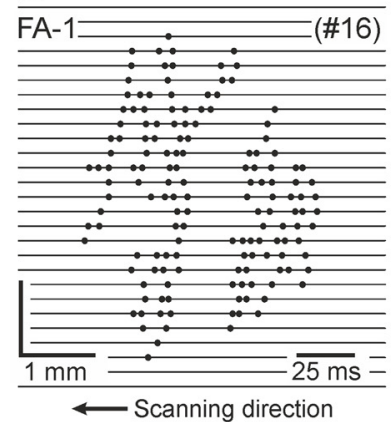

D

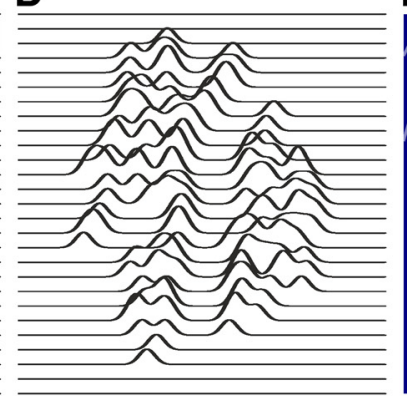

E

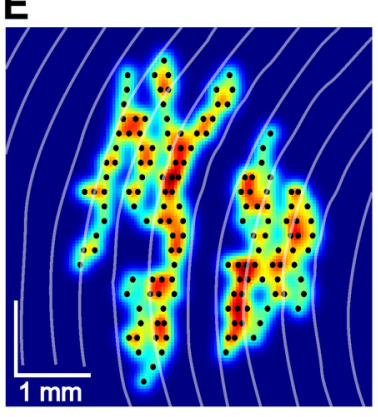

Figure 1. Experimental setup, stimuli, and basic approach. $\boldsymbol{A}$, Stimulating surface with raised dots for mapping receptive field sensitivity topography of first-order tactile neurons. $\boldsymbol{B}$, The surface was wrapped around a transparent drum and a custom-built robotic device controlled the drum's rotation and position. Inset shows schematically one of the raised dots. $\boldsymbol{C}$, Two-dimensional SEP for an exemplar FA-1 neuron (\#16) obtained during one drum rotation in proximal-distal direction at 30-mm/s tangential speed. Each point represents the occurrence of an action potential. The thin horizontal lines show the paths of dots that scanned fingertip. $\boldsymbol{D}$, Receptive field sensitivity map obtained after convolving spike events in $\boldsymbol{C}$ with a one-dimensional kernel $(S D=0.1 \mathrm{~mm}) . \boldsymbol{E}$, Color-coded sensitivity map obtained after convolving the same spike events with a two-dimensional kernel (SD=0.1 mm). For reference, the superimposed small black dots represent the action potentials of the SEP shown in $\boldsymbol{C}$. The white lines mark the grooves between the fingerprint (papillary) ridges.

interdigitating subfields might determine the limit of the spatial resolution rather than the much greater distance between their receptive field centers as traditionally thought (Johnson and Phillips, 1981; Phillips et al., 1983; Van Boven and Johnson, 1994; Weber et al., 2013). In fact, the spatial accuracy in geometric tactile processing during object manipulation (Pruszynski et al., 2018) and in certain psychophysical tasks (Loomis and Collins, 1978; Loomis, 1979; Wheat et al., 1995; Hollins and Bensmaïa, 2007) can exceed that predicted by the ShannonNyquist sampling theorem based on the distance between receptive field centers. However, according to our hypothesis, the intrinsic spatial resolution of the peripheral tactile apparatus would depend not only on the density of the subfields in the skin but also on the size of the skin area subtended by each of them, where a smaller size would allow for detection of finer spatial inhomogeneities. Although the spatial acuity of the subfields of the FA-1 and SA-1 neurons has not yet been quantified, it can be assumed to approach the dimension of individual fingerprint ridges. First, the receptor organs responsible for the subfields of these neuron types are directly associated with individual papillary ridges (Cauna, 1954; Halata, 1975) and could therefore measure deformations of a single ridge. Second, a ridge can be deflected largely independently of its neighbors (Johansson and LaMotte, 1983; LaMotte and Whitehouse, 1986; Lee et al., 2020). Furthermore, human studies suggest that perception of spatial details improves with reduced width of papillary ridges (Peters et al., 2009) and relatedly increased density of ridge-associated receptor organs (Dillon et al., 2001).

Here, we quantified the spatial acuity of human FA-1 and SA-1 neurons' subfields and examined how robustly the subfield layout structures a neuron's response in the spatial domain when tactile stimuli slide across the fingertip at different speeds. The neurons were stimulated with small raised dots on a flat background surface moving tangentially across the receptive field at speeds representative of those naturally used in tactile pattern discrimination tasks (15, 30, and $60 \mathrm{~mm} / \mathrm{s}$; Lederman, 1974; Vega-Bermudez et al., 1991; Boundy-Singer et al., 2017; Olczak et al., 2018). We also analyzed effects of different scanning directions, like back and forth exploratory movements with a fingertip. With subfield receptors connected to papillary ridges and frictional forces generating direction-dependent shear deformations of the fingerprint within the contact area (Delhaye et al., 2016), we anticipated some directional effects on the relative positions of the subfields within a neuron's receptive field.

\section{Materials and Methods}

Participants and general procedure

Twelve healthy humans, 20-30 years of age (six females), participated after providing written informed consent in accordance with the Declaration of Helsinki. The Umeå University ethics committee approved the study.

Each subject reclined comfortably in a dentist's chair with the right upper arm abducted $\sim 30^{\circ}$, the elbow extended to $\sim 120^{\circ}$, and the hand supinated. A vacuum cast, supported by a metal frame, immobilized the forearm, and Velcro strips around the wrist provided additional fixation. To stabilize fingertips, we glued the nails to plastic holders firmly attached to the frame that also supported a robot that controlled the tactile stimulation (Birznieks et al., 2001).

Action potentials from single first-order tactile neurons terminating in the glabrous skin of the distal segment of the index, long or ring finger were recorded with tungsten electrodes (Vallbo and Hagbarth, 1968) percutaneously inserted into the right median nerve at the mid-level of the upper arm. Isolated neurons were classified as FA-1, SA-1, FA-2, and SA-2, according to previously described criteria (Vallbo and Johansson, $1984)$. We focused on FA-1 neurons $(N=23)$ and SA-1 neurons $(N=11)$ whose well-defined cutaneous receptive fields are made up of a number of subfields (Johansson, 1978; Phillips et al., 1992; Pruszynski and Johansson, 2014).

\section{Tactile stimuli}

We analyzed the neurons' responses elicited by a stimulus pattern that contained raised dots on a flat background moving tangentially across the receptive field (Fig. $1 A$ ). The dots were 0.45 -mm-high truncated cones with a flat $0.4 \mathrm{~mm}$ diameter top and a base diameter of $0.7 \mathrm{~mm}$ (Fig. $1 B$, inset). The stimulus pattern was produced via a standard photo-etching technique using a photosensitive nylon polymer (Toyobo EF 70 GB, Toyobo Co, Ltd.) and wrapped around a transparent rotating drum (Fig. 1B). A custom-built robotic device controlled the rotation speed of the drum and kept the normal contact force between the stimulus pattern and the receptor-bearing fingertip constant at $\sim 0.4 \mathrm{~N}$ (for details, see Pruszynski and Johansson, 2014). This force was chosen because it falls within the range that humans use to manually explore 
surfaces (Lederman, 1974; Gamzu and Ahissar, 2001; Smith et al., 2002; Olczak et al., 2018). A video camera mounted in the transparent drum was used to position the stimulus pattern with reference to the location of the neuron's receptive field on the fingertip as previously described (Johansson and Vallbo, 1980).

The stimulus surface included stimuli used to generate a sensitivity map of the neuron's receptive field (Fig. 1A). The layout of the field mapping dots was designed to generate a field sensitivity map for each drum revolution based on one dot stimulating the neuron at the time. Forty-one dots were equally distributed along the extent of the stimulation surface in the movement direction (length $=312 \mathrm{~mm}$ ), defined as the $x$-direction. In the perpendicular direction, defined as the $y$-direction, the dots were equally spaced on the $8 \mathrm{~mm}$ wide zone. Thus, the dots moved over the skin in separate tracks spaced $0.2 \mathrm{~mm}$ apart. Overall, the dots were spaced at least $7 \mathrm{~mm}$ apart to minimize interactions between neighboring dots on a neuron's response (Phillips et al., 1992). The array contained four additional dots that were evenly spaced in the $x$-direction and located in the center of the mapping zone in the $y$-direction. These dots, which nominally moved across the center of the neuron's receptive field, could be used to control the spatial alignment of action potentials in the movement direction.

\section{Experimental design and statistical analysis \\ Stimulation protocol}

For each neuron, the field mapping zone was moved over its receptive field in the proximal-distal direction of the finger at a speed of $30 \mathrm{~mm} / \mathrm{s}$ for four drum revolutions. Thereafter, the drum was laterally repositioned to expose the receptive field to another stimulation pattern containing raised elements for 15 drum revolutions in the same direction and movement speed (data not shown). For neurons with stable enough recording of unitary action potentials, the corresponding protocol was then run at a speed of 60 and $15 \mathrm{~mm} / \mathrm{s}$ (10 FA-1s and 6 SA-1s). For neurons with still discriminable unitary action potentials, we then ran the above scheme with drum rotations at $30 \mathrm{~mm} / \mathrm{s}$ in the opposite direction, i.e., the stimulus pattern now moved in the distal-proximal direction (8 FA-1s and 3 SA-1s). For analysis of the effect of scanning direction on receptive field sensitivity topography we also included data for proximal-distal and distal-proximal stimulation at $30 \mathrm{~mm} / \mathrm{s}$ gathered in a previous series of experiments but not analyzed direction-wise (8 FA-1s and 3 SA-1s; Pruszynski and Johansson, 2014).

\section{Data processing and analysis}

The nerve signal, the instantaneous position of the stimulus surface recorded via a drum shaft decoder (AC36, Hengstler $\mathrm{GmbH}$ ) providing a resolution of $3 \mu \mathrm{m}$, and the contact force were digitally sampled at $19.2,2.4$, and $0.6 \mathrm{kHz}$, respectively (SC/ZOOM, Department of Integrative Medical Biology, Umeå University). Unitary action potentials were detected online based on spike morphology and verified for each action potential off-line (Edin et al., 1988).

From action potentials recorded during the second, third, and fourth drum revolution, we constructed separate two-dimensional spatial event plots (SEPs; Johnson and Lamb, 1981) of the neuron's receptive field based on the position of the stimulating dot at each evoked action potential (Fig. 1C). Since the dots were distributed along the direction of motion of the stimulus pattern ( $x$-direction), the instantaneous $x$-coordinate of the stimulating dot was offset based on its known $x$-coordinate. The $y$-position was defined by the $y$-coordinate of the track (one out of 41) in which the stimulating dot moved. We omitted data from the first drum revolution because visual inspection revealed that the tendency to creep deformation of the fingertip that would distort the construction of SEPs was most pronounced during the first revolution.

To render a smooth receptive field map, we convolved with a Gaussian function SEPs obtained from each drum revolution within an 8 by $8 \mathrm{~mm}$ window centered on the centroid of the spike activity. Figure $1 D$ illustrates a receptive field sensitivity map obtained by convolving a neuron's spike traces with a kernel width of $0.1 \mathrm{~mm}$, and Figure $1 E$ shows a color-coded map generated with a corresponding two-dimensional Gaussian where brighter colors indicate higher spatial density of action potentials. For each SEP, mean firing rate was calculated as the number of spikes evoked within the $8 \times 8$-mm window divided by the duration of the stimulus dot within this window. Peak firing rate was defined as the reciprocal of the shortest, stimulus-evoked interspike interval observed in a SEP.

Our estimates of the spatial acuity of a neuron's subfields and how its receptive field sensitivity topography can be affected by scanning speed and direction relied on assessment of similarity between maps based on pairwise cross-correlations of SEPs after convolution with Gaussians of different widths defined in the spatial domain. By convolving each SEP with 21 different kernels with logarithmically spaced standard deviations (SDs) in the range of $0.02-0.33 \mathrm{~mm}$, we gradually simulated increased noise on the positions of the action potentials, which increasingly blurred the representation of the field sensitivity topography. Our approach is analogous to methods previously used to assess the similarity of pairs of individual spike trains obtained under different experimental conditions but when represented in the temporal domain (Schreiber et al., 2003; Fellous et al., 2004; Vázquez et al., 2013; Pruszynski and Johansson, 2014).

To account for skin warping in the analysis of effects of scanning direction on the receptive field sensitivity topography, we used iterative cross-correlation to define parameters for transforming the map obtained in the distal-proximal scanning direction to best resemble that obtained in the proximal-distal direction. The parameter values used were those that generated the maximum correlation coefficient after \pm 4 $\mathrm{mm}$ stretching/compression of the map $(8 \times 8 \mathrm{~mm})$ in increments of 0.2 $\mathrm{mm}$ in the scanning direction and in its perpendicular direction, and rotation of the map $\pm 20^{\circ}$ in $2^{\circ}$ increments, taking into account every possible combination in the matrix.

To visually relate a neuron's subfield layout to the arrangement of the papillary ridges, we overlaid the sensitivity map on manual tracings of the papillary grooves within the appropriate skin surface based on a still image taken from the recorded video; the spatial acuity of our video monitoring system and its temporal resolution (1 frame per $40 \mathrm{~ms}$ ) were not sufficient for analysis of time-varying correlations between spike events and deformation changes of individual ridges caused by the dot stimulus. To help fine-tune the placement of the map under the assumption that the distribution of the clusters of spikes in the SEP representing subfields was structured by the ridge pattern, we cross-correlated the SEP convolved with a Gaussian kernel $(\mathrm{SD}=0.1 \mathrm{~mm})$ and the traced ridge pattern. Each ridge was represented by a half cosine cycle specified between $-\pi / 2$ and $\pi / 2$ along the track for each stimulation dot.

We calculated an average of the ridge width (RW) in the receptive field by measuring the length of a line oriented such that it transversely crossed five ridges centrally in the field. We also recorded the orientation of this line with reference to the scanning direction $(\alpha)$. Although the basic types of fingerprints are arches, radial loops, ulnar loops, and whorls (Galton, 1892), when a small area corresponding to the size of the current receptive fields is considered, the ridges were usually quite parallel (see Results). We estimated the distance by which the leading edge of the dot stimulus traveled across a ridge by considering the ridge orientation relative to the scanning direction. The shortest distance occurs when a ridge is oriented perpendicular to the scanning direction, while the distance gradually increases when the ridge orientation becomes increasingly oblique relative to the scanning direction. The increase of the distance as a function of $\alpha$ was calculated as RW/cosine $(\alpha)$-RW.

\section{Statistical analysis}

All cross-correlation analyses were made with the Normalized 2-D cross-correlation in MATLAB R2019b (https://www.mathworks.com/ help/images/ref/normxcorr2.html). Correlation coefficients were Fisher transformed into $\mathrm{Z}$ scores when performing parametric statistics and in estimating average values they were then converted back to correlation coefficients. Correlation values are reported as coefficients of determination $\left(R^{2}\right)$. Effects of the experimental factors on neural response variables were assessed using two-tailed $t$ test for independent samples by groups and two-way mixed-design ANOVAs with neuron type (FA-1 and SA1 ) as a between-group effect. We used the Tukey's HSD test for post hoc comparisons. All statistical tests were deemed significant if $p<0.05$. 

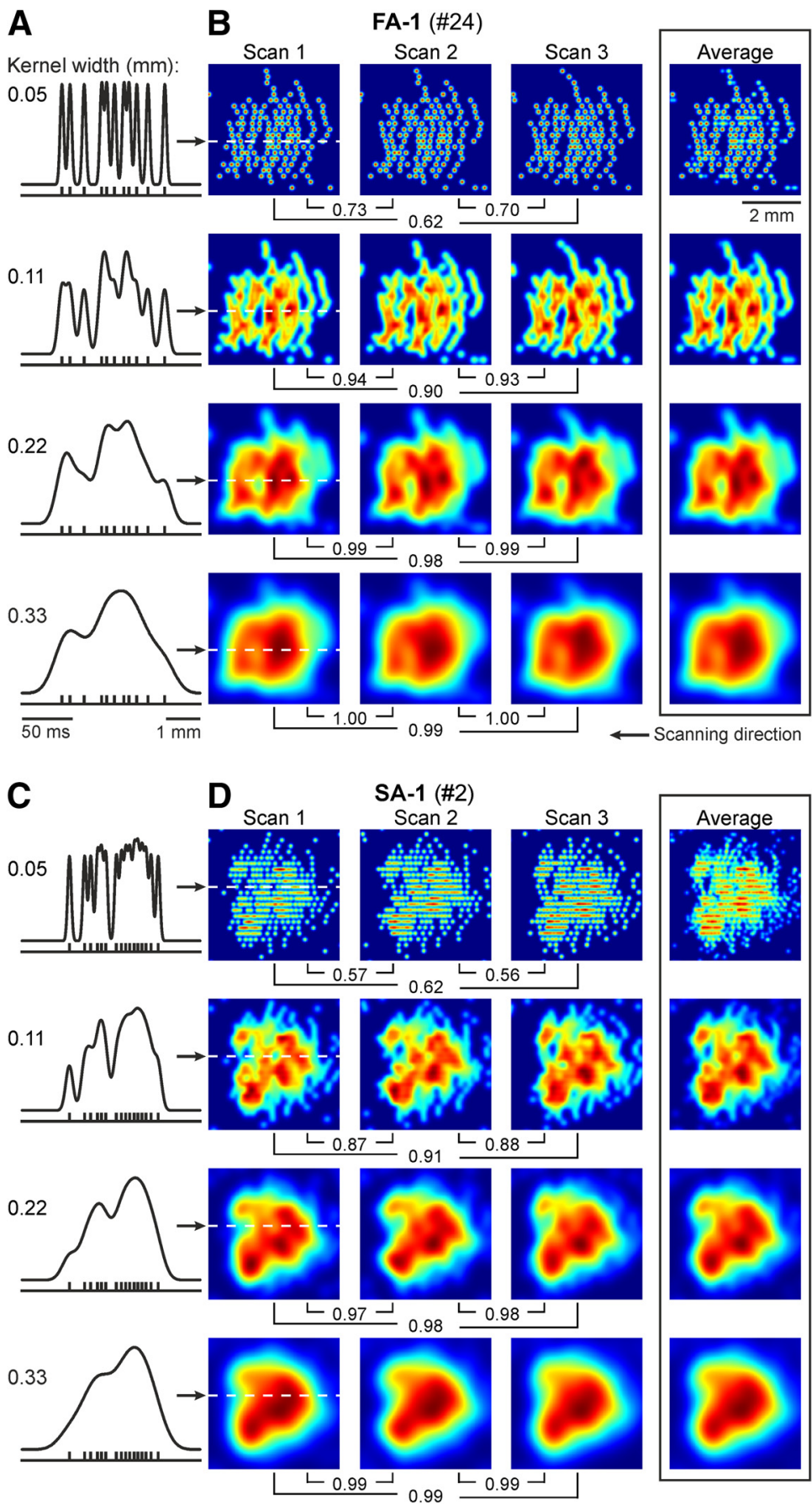

Figure 2. Effect of kernel width on the receptive field map. $\boldsymbol{A}$, The different panels show, for an exemplar FA-1 neuron (\#24), one spike train (bottom trace) elicited by one of the stimulus dots when passing along its track over the receptive field (dashed white line in $\boldsymbol{B}$ ) and this train convolved with four of the 21 different kernels used ( $S D=0.05,0.11,0.22$, and $0.33 \mathrm{~mm}$; top trace). $\boldsymbol{B}$, Sensitivity maps of the same neuron obtained by convolving the SEPs generated during each of the three scans (scans 1-3) with the kernel widths shown in $\boldsymbol{A}$. The rightmost sensitivity maps represent, for each kernel width, the average of the three maps. The numbers indicate $R^{2}$ values of correlated pairs of maps. $\boldsymbol{C}, \boldsymbol{D}$, Data from an exemplary SA-1 neuron (\#2) shown in the same format as in $\boldsymbol{A}, \boldsymbol{B}$. $\boldsymbol{A}-\boldsymbol{D}$, Neurons scanned at $30 \mathrm{~mm} / \mathrm{s}$ in the proximal-distal direction.

Unless otherwise stated, reported point estimates based on sample data refer to mean $\pm 1 \mathrm{SD}$.

\section{Results}

We present the results in four sections. First, we estimate the spatial acuity of FA-1 and SA-1 neurons' subfields and provide evidence suggesting that the acuity matches the dimension of an individual fingerprint ridge. Second, we analyze the similarity of a neuron's receptive field maps obtained across repeated mappings and address heterogeneity among neurons regarding the subfield layout. Third, we test how well a neuron's field sensitivity topography is maintained at different scanning speeds $(15,30$, and $60 \mathrm{~mm} / \mathrm{s})$. Fourth, we investigate the consistency of the receptive field sensitivity topography across different stimulation directions by comparing results from scans in the proximal-distal and distal-proximal direction.

\section{Spatial acuity of subfields}

To estimate with which acuity a neuron's subfield layout structures its response in the spatial domain, we first generated a set of receptive field maps by convolving the SEP obtained at each scan with a two-dimensional Gaussian function at 21 different kernel widths with standard deviations increasing from 0.02 to 0.33 $\mathrm{mm}$ (Fig. 2A,C). Thus, we simulated gradually increased noise on the positions of the action potentials, which increasingly blurred the representation of the sensitivity topography of the receptive field (Fig. 2B,D). We then calculated the pairwise two-dimensional cross-correlation between the three maps which resulted in three correlations per kernel width and stimulation condition (scanning speed and direction; Fig. $2 B, D)$.

As illustrated in Figure $3 A$, for all 34 neurons stimulated at $30 \mathrm{~mm} / \mathrm{s}$ in the proximal-distal direction the correlation between the three maps increased as a function of kernel width (solid lines). The low correlations obtained with the narrowest kernels arose because the spike jitter between repetitions of the same stimulus tended to be greater than the kernel width. With gradually wider kernels, the correlation increased steeply up to around 0.1-mm width and then remained high as the maps became more Gaussian-shaped and moved toward having a single point of maximum sensitivity (Fig. $2 B, D)$. The kernel width at this breaking point $(\sim 0.1 \mathrm{~mm})$ provided an initial estimate of the spatial sensitivity of a neuron's subfield arrangement since additional spatial filtering that attenuated the sensitivity topography of the receptive field did not substantially increase the correlation.

To further assess the reliability of this estimate, we compared the mean value of the correlations between the three empirical maps as a function of kernel width with the corresponding mean of pairwise correlations between each of the three empirical maps and the same map rotated $180^{\circ}$ (three correlations for each kernel width). The rotation confused the internal sensitivity topography of a neuron's receptive field while maintaining its generally oval shape, orientation, and size. As expected, compared with the correlation between the empirical maps, this 
confusion regarding the subfield arrangement resulted in a slower increase in correlation with increased kernel width for widths up to $\sim 0.1 \mathrm{~mm}$ (Fig. $3 A$, dashed lines). We calculated the difference between the mean values for the correlations between the empirical maps and the correlations that included $180^{\circ}$ map rotation as a function of the kernel width and used the kernel width where the difference was maximal as a point estimate of a neuron's spatial sensitivity with respect to its subfield arrangement (Fig. $3 B, C$ ). For neurons scanned at $30 \mathrm{~mm} / \mathrm{s}$ in the proximal-distal direction, the estimated subfield acuity was on average $0.081 \pm 0.025 \mathrm{~mm}$ (mean $\pm \mathrm{SD}, N=34$ ) and did not reliably differ between neuron type $\left(t_{(32)}=\right.$ 0.73 ; $p=0.47$; $t$ test for independent samples by groups).

Given that the receptor organs of FA-1 and SA-1 neurons are associated with individual papillary ridges, we sought to relate neurons' subfield acuity to the dimensions of the ridges within their receptive fields. For this, we expressed a neuron's subfield sensitivity profile with a sinus function, using the fact that a basic cosine cycle specified between $-\pi$ and $\pi$ is very similar to a Gaussian function within \pm 2.5 SDs $\left(R^{2}=0.996\right)$. Hence, in sinusoidal terms, the spatial sensitivity was $0.41 \pm 0.12 \mathrm{~mm}$ averaged across the neurons (i.e., five times that expressed as kernel width; Fig. $3 C$, upper abscissa). Measurements within the neurons' receptive fields indicated that the width of the papillary ridges was similar: $0.47 \pm 0.10 \mathrm{~mm}$ $(N=33$; video image was missing for one SA-1 neuron). These results suggested that the spatial acuity of the subfields basically matched the width of an individual ridge. Likewise, inspection of the receptive field maps gave the impression that the dimension of individual subfields, representing clustering of action potentials, often corresponded to the width of a ridge and in some cases seemed to be even smaller (Fig. $1 E$, also see Figs. $5 B, 6 A$ ).

We asked whether the spatial acuity of a neuron's subfields is directly linked to the width of the ridges in its receptive field. We addressed this with a multiple linear regression that used the variability between neurons in estimated spatial acuity as the dependent variable and ridge width as one independent variable. A second independent variable dealt with the possibility that the subfields had a farther extent and thus a poorer spatial selectivity for stimuli moving along the ridges compared with mainly across the ridges. The variation in the path of the stimulation dots in this respect was significant among our neurons. That is, for some the dots moved mainly across the ridges and for others along the ridges as well as in the directions in between (Figs. 5B,6A). Referring to straight across the papillary ridges centrally in the receptive field, the tracks of the stimulation dots were approximately uniformly distributed in the range between $1^{\circ}$ and $89^{\circ}(\mathrm{Q} 1-$ $\mathrm{Q} 3=13-62^{\circ}$, median $\left.=42^{\circ} ; N=33\right)$. Specifically, this second independent variable indicated the increase in distance that

A
B
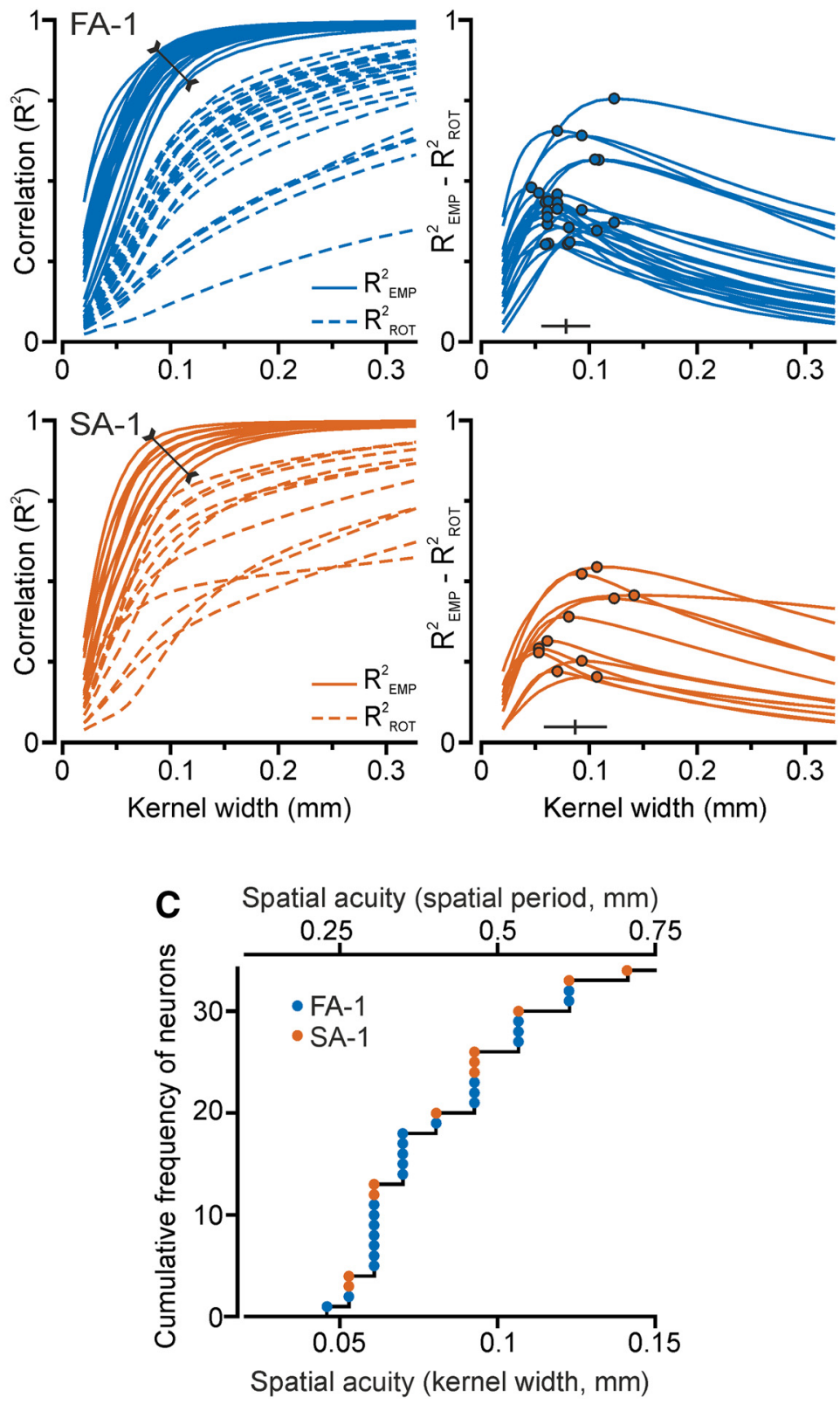

Figure 3. Spatial acuity when scanned at $30 \mathrm{~mm} / \mathrm{s}$ in the proximal-distal direction. $\boldsymbol{A}$, Superimposed curves show, for individual neurons (23 FA-1s, $11 \mathrm{SA}-1 \mathrm{~s}$ ), mean values of pairwise correlations between the empirical maps obtained during the three scans (solid lines, $R_{\text {EMP) }}^{2}$ and of correlations between each of the three empirical maps and the same map rotated by $180^{\circ}$ (dashed lines, $R_{\text {ROT }}^{2}$ ) as a function of kernel width. The slanted line with arrowheads at the ends, centered on 0.1-mm kernel width, roughly marks the breaking point where further spatial filtering did not substantially increase the correlations between the empirical maps. $\boldsymbol{B}$, Difference between correlations among the empirical maps and those involving $180^{\circ}$ map rotation for individual neurons as a function of kernel width. The filled circles indicate the point of maximum difference for each neuron and the horizontal bar indicates mean $\pm S D$ across neurons of the kernel width at this point. C, Distribution across neurons of the estimated spatial acuity represented as the kernel width yielding the maximum difference (bottom abscissa) and as a sinusoidal spatial period (top abscissa). Clustering of data points at different abscissa values results from the kernel widths used for convolving with the spike trains (see Materials and Methods).

the stimulus interacted with the ridges depending on the obliqueness of their orientation relative to the scanning direction (see Materials and Methods). A reliable regression equation was found $\left(R^{2}=0.27, F_{(2,30)}=5.67, p=0.008\right)$ although the model did not factor in variations in the orientation of the ridges within the field caused by their curvature tendencies. Both, ridge width and distance increase were significant 


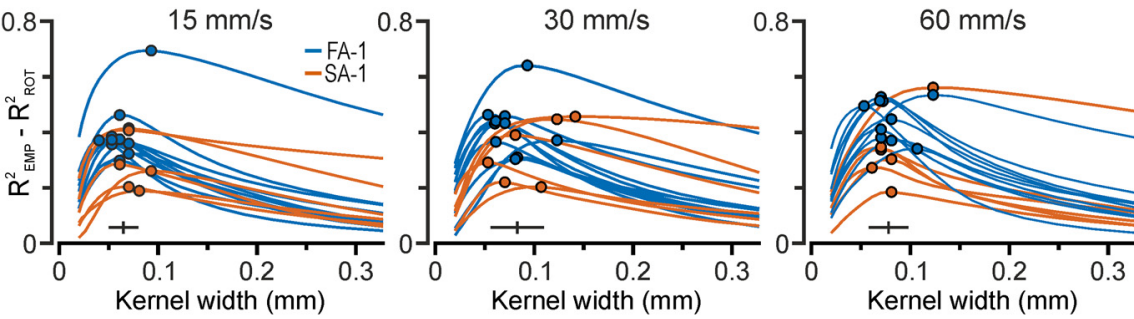

Figure 4. Spatial acuity at different scanning speeds. Difference between correlations among the empirical maps and those involving $180^{\circ}$ map rotation for individual neurons mapped at all three scanning speeds $(15,30$, and $60 \mathrm{~mm} / \mathrm{s})$ as a function of kernel width. Same format as Figure $3 B$.

predictors of spatial acuity $(\beta=0.49, p=0.005$ and $\beta=0.38$, $p=0.027$, respectively). The predicted acuity expressed as spatial period was equal to $0.08+0.63 \times(\mathrm{RW})+0.08 \times$ (increased distance), all measures in millimeters. Thus, the spatial period representing a neuron's subfield acuity increased by $0.063 \mathrm{~mm}$ for each $0.1-\mathrm{mm}$ increase in ridge width. However, it only increased by $0.008 \mathrm{~mm}$ for each 0.1 $\mathrm{mm}$ increase in the stimulation distance along the ridges, which suggests that the spatial selectivity of the subfields was similar for stimuli moving along a ridge as for stimuli moving across a ridge. Overall, these results are consistent with the idea that a subfield essentially records tactile events localized to a limited segment of an individual ridge.

The effect of scanning speed $(15,30$, and $60 \mathrm{~mm} / \mathrm{s})$ on the spatial acuity was investigated for 10 FA-1 and 6 SA-1 neurons stimulated in the proximal-distal direction. For the 15- and the$60 \mathrm{~mm} / \mathrm{s}$ scanning speeds, the effect of kernel width on the correlations between the empirical maps and those involving $180^{\circ}$ map rotation was similar to that for $30 \mathrm{~mm} / \mathrm{s}$ (Fig. 4). We found an effect of speed on the spatial acuity $\left(F_{(2,28)}=5.00, p=0.014\right)$, the kernel width tended to be smaller at $15 \mathrm{~mm} / \mathrm{s}(0.065 \pm 0.015$ $\mathrm{mm})$ than at $30 \mathrm{~mm} / \mathrm{s}(0.083 \pm 0.027 \mathrm{~mm} ; p=0.01$, Tukey's HSD post hoc test $)$ and $60 \mathrm{~mm} / \mathrm{s}(0.078 \pm 0.020 \mathrm{~mm} ; p=0.08)$ and did not statistically differ between 30 and $60 \mathrm{~mm} / \mathrm{s}(p=0.63)$. There was no effect of neuron type $\left(F_{(1,14)}=0.92 p=0.35\right)$ and no interaction effect between speed and neuron type $\left(F_{(2,28)}=1.35\right.$, $p=0.27$.

In sum, the spatial sensitivity of the subfield arrangement of the FA-1 and the SA-1 neurons corresponded to kernel widths around $0.1 \mathrm{~mm}$ and slightly below, it was barely affected by scanning speed and expressed as spatial period it matched the width of single papillary ridges. The remainder of the results section is based on analyses where we consistently used receptive field maps obtained with a kernel width of $0.1 \mathrm{~mm}$. Note that none of our conclusions were qualitatively altered with corresponding analyses based on kernel widths identified for each individual neuron.

\section{Consistency and heterogeneity of neurons' subfield arrangement}

A neuron's receptive field maps obtained at the three consecutive scans at a given speed and direction were very similar (Fig. 2B,D). For scans at $30 \mathrm{~mm} / \mathrm{s}$, the mean correlation for the three pairwise cross-correlations obtained for the individual neurons (Fig. 5A, filled circles) averaged 0.90 (mean $R^{2}$; median $\left.=0.89\right)$ across the 34 neurons and did not differ reliably between neuron type $\left(t_{(32)}=1.95 ; p=0.06 ; t\right.$ test for independent samples by groups). The variability in $R^{2}$ values across the pairwise correlations was small (Fig. 5A, gray area around top curve).

To provide a reference for the correlation observed across repeated mappings with regard to the significance of the subfield layout, first we used the pairwise correlations between each of the empirical maps and the same map rotated by $180^{\circ}$ (Fig. 5A, filled squares). As indicated above, these correlations involved disruption of the subfield layout while being modestly affected by the oval overall shape of the receptive field and its orientation. Second, we cross-correlated each of the three empirical maps with each of the maps obtained at the corresponding scan of all other neurons $(3 \times 33=99$ correlations per neuron; Fig. $5 A$, open circles). This "shuffling" would likely yield worse correlations because the maps would also be sensitive to the principal orientation as well as to the overall size of the receptive field.

In Figure 5A, the neurons are ranked along the abscissa based on the difference between the correlation of the empirical maps and the correlation involving $180^{\circ}$ map rotation. Averaged across all neurons, the latter correlation was markedly lower than the correlation between the empirical maps (mean $R^{2}=0.52$ vs 0.90 ). However, the difference varied substantially between neurons (Fig. 5A, vertical distance between the filled circle and squares). Neurons with the smallest differences (subfield arrangement least sensitive to receptive field rotation), usually had quite complex receptive fields but with a noticeable $180^{\circ}$ rotational symmetry, or occasionally a field with essentially only one highly sensitive zone (Fig. 5B, top row). Neurons with intermediate differences usually showed complex multifocal receptive fields (Fig. 5B, middle row) and those with the largest difference typically had very patchy receptive fields with widely spread subfields (Fig. 5B, bottom row). Figure $5 C$ shows the receptive fields displayed in Figure $5 B$ arbitrarily projected on the fingerprint when a fingertip contacts a flat surface. Note that the receptive field of an individual neuron can occupy a significant part of the contact area. A two-way mixed design ANOVA applied to the difference in the correlations involving the empirical maps and those involving map manipulations $\left(180^{\circ}\right.$ rotation, shuffling) indicated a main effect of the map manipulation $\left(F_{(1,32)}=38.39, p<0.0001\right)$ but not of neuron type $\left(F_{(1,32)}=0.23, p=0.63\right)$ and no significant interaction $\left(F_{(1,32)}=0.55, p=0.46\right)$. The field shuffling yielded a weaker correlation than the $180^{\circ}$ rotation. The differential effect of the $180^{\circ}$ rotation and the shuffling could markedly vary between neurons (Fig. 5A) where neurons with widely scattered subfields were similarly affected.

For the 16 neurons scanned at all three speeds $(15,30$, and 60 $\mathrm{mm} / \mathrm{s}$ ) in the proximal-distal direction, scanning speed affected the correlations between the empirical maps $\left(F_{(2,28)}=58.76\right.$, $p<0.0001)$. The average $R^{2}$ of the empirical maps was $0.94,0.90$, and 0.86 at 15,30 , and $60 \mathrm{~mm} / \mathrm{s}$, respectively (Fig. $5 D$ ). There was no main effect of neuron type $\left(F_{(1,14)}=0.09, p=0.77\right)$ or interaction effect between speed and neuron type $\left(F_{(2,28)}=1.29\right.$, $p=0.29)$. As with $30 \mathrm{~mm} / \mathrm{s}$, the variability in the pairwise correlations at 15 and $60 \mathrm{~mm} / \mathrm{s}$ was small. For 15 and $60 \mathrm{~mm} / \mathrm{s}$, the effect of the map manipulations was like that described above for $30 \mathrm{~mm} / \mathrm{s}$ (Fig. 5D). That is, for the neurons scanned at all three speeds, a three-way mixed design ANOVA failed to indicate an 

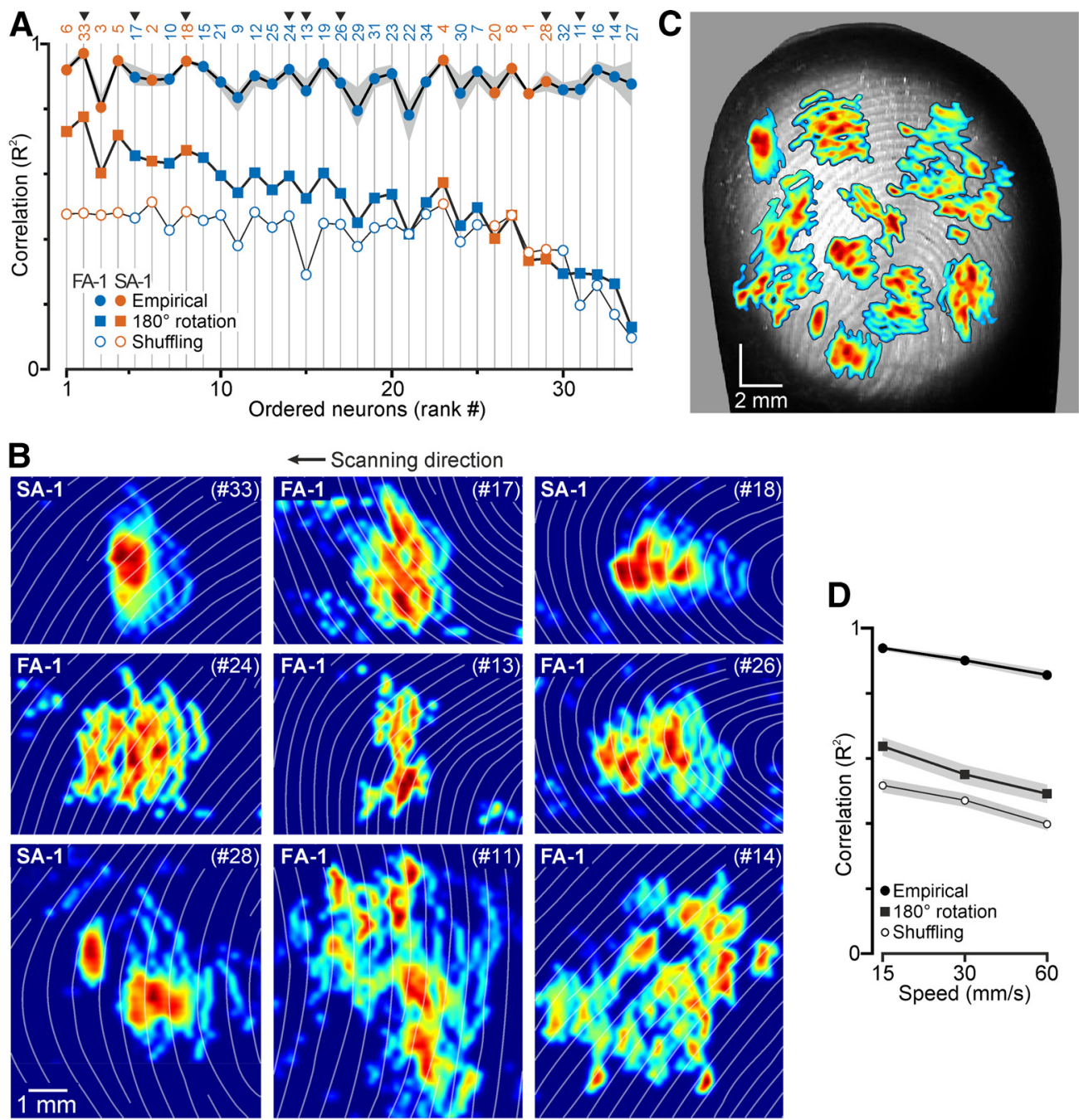

Figure 5. Consistency and heterogeneity of neurons' subfield layout. $\boldsymbol{A}$, Filled circles joined by the top curve show, for each neuron (23 FA-1s, 11 SA-1s), the mean value of the three correlations between the maps of the three scans at $30 \mathrm{~mm} / \mathrm{s}$ in the proximal-distal direction. The gray shading indicates the range of these correlations. Correspondingly, filled squares show the mean value of the correlations involving $180^{\circ}$ map rotation, and hollow circles show the mean correlation between each of the three empirical maps and each of the corresponding maps of all other neurons ("shuffling"). Numbers at the top indicate the identification number for each neuron used throughout the article, and arrowheads indicate neurons featured in $\boldsymbol{B}$. Neurons have been ranked along the abscissa as a function of increasing difference between the correlations among the empirical maps and those involving $180^{\circ}$ map rotation. $\boldsymbol{B}$, Examples of receptive field sensitivity maps of neurons with small, intermediate and large difference (top, middle, and bottom panels, respectively) obtained by scans at $30 \mathrm{~mm} / \mathrm{s}$ in the proximal-distal direction; average map across the three scans is shown. The white lines indicate the grooves between the papillary ridges. $\boldsymbol{C}$, Receptive fields shown in $\boldsymbol{B}$ projected on a fingerprint photographed through a flat glass plate when contacted by a fingertip with approx. $0.5-\mathrm{N}$ normal force. The fields have been arbitrarily placed on the contact surface. In reality, there is a massive overlap of such fields within the contact area. With an innervation density of $\sim 140 \mathrm{FA}-1$ and $\sim 70$ SA-1 neurons per $\mathrm{cm}^{2}$ (Johansson and Vallbo, 1979a), fields belonging to $\sim 500$ neurons would occupy the displayed contact area $\left(\sim 2.5 \mathrm{~cm}^{2}\right)$. D, Mean $R^{2}$ values from $A$ as a function of scanning speed $(15,30$, and $60 \mathrm{~mm} / \mathrm{s})$. The gray shading indicates standard error of the mean $(N=16)$.

effect of speed and neuron type on the difference in mean correlations between the empirical maps and the correlations involving the field manipulations $\left(F_{(2,28)}=2.06, p=0.16\right.$ and $F_{(1,14)}=$ $1.94, p=0.18$, respectively), while map manipulation had a main effect $\left(F_{(1,14)}=39.00, p<0.0001\right)$ with a greater difference with shuffling than with $180^{\circ}$ map rotation. There were no significant interaction effects between these factors.

In sum, these results show that the sensitivity topography of the receptive fields is well conserved across consecutive scans regardless of speed but can be quite heterogeneous across neurons.

Conservation of receptive field sensitivity topography across scanning speeds

Based on data from neurons scanned at all three speeds in the proximal-distal direction, we asked to what extent a neuron's subfield layout is maintained across scanning speeds. In this analysis we used an average of the three maps obtained with each scanning speed constructed with the $0.1-\mathrm{mm}$ kernel width.

Visual inspection of the maps indicated that a neuron's subfield stood out with a similar layout at all speeds (Fig. 6A). However, decreases in speed resulted in an increased maximum spike density in the subfields, which is consistent with previous results regarding the effect of speed on the number of action potentials of a neuron's SEP (Phillips et al., 1992). A two-way ANOVA verified that speed influenced the number of action potentials $\left(F_{(2,28)}=61.5, p<0.0001\right)$ but not neuron type $\left(F_{(1,14)} \leq 2.55, p \geq 0.13\right)$. Considering firing rates, the mean as well as the peak firing rate increased with increasing speed $\left(F_{(2,28)}=88.9, p<0.0001\right.$ and $F_{(2,28)}=17.4$, $p<0.0001$, respectively), with no significant effect of neuron type $\left(F_{(1,14)} \leq 2.55, p \geq 0.13\right.$ in both instances). Averaged across the three scans and all 16 neurons, the mean rate was $11 \pm 5,21 \pm 7$, and $26 \pm 9 \mathrm{~Hz}($ mean $\pm \mathrm{SD})$ at 15,30 , and 60 

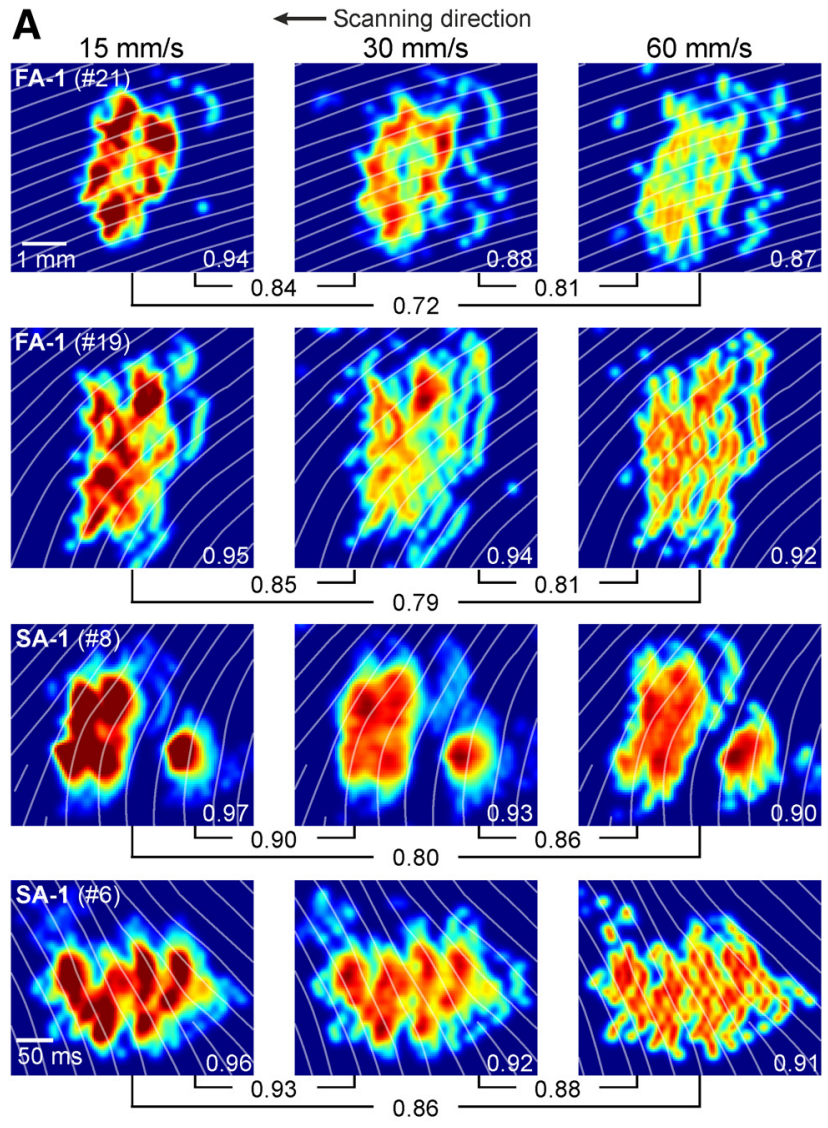

B

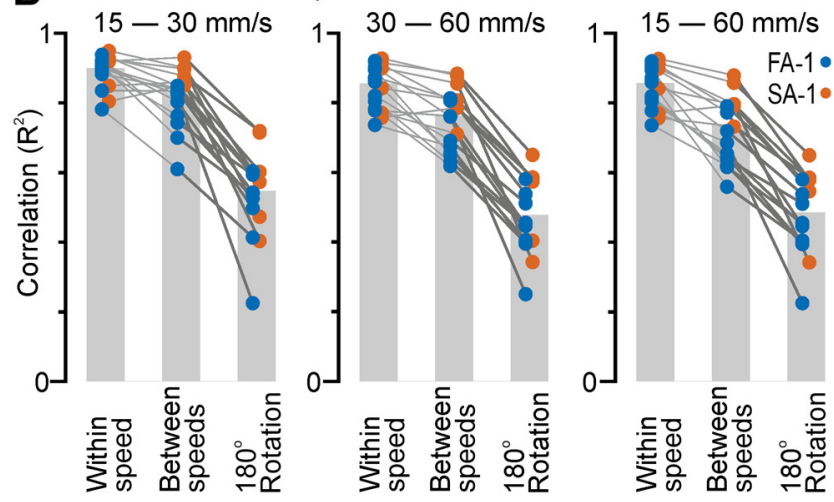

Figure 6. Effect of scanning speed on sensitivity topography. $A$, Sensitivity maps obtained at $15-, 30-$, and $60-\mathrm{mm} / \mathrm{s}$ scanning speed for two exemplar neurons of each type (FA-1 and $S A-1)$, average map across the three scans is shown. The white lines mark the grooves between the papillary ridges. The numbers underneath the maps indicate $R^{2}$ values of correlated pairs of maps (between-speed correlations) and the numbers on the maps indicate, for each map, the mean $R^{2}$ of the correlations between the empirical maps obtained during the three scans (within-speed correlations). $\boldsymbol{B}$, For each speed combination and neuron, symbols show: (1) average correlation between the three empirical maps for the speed that showed the lowest correlation ("within speeds"); (2) pairwise correlation values between average maps obtained with the respective speed ("between speeds"); (3) average correlation between each of the empirical maps and the same map rotated $180^{\circ}$ for maps involved in respective speed combination ("180 rotation"). Lines join symbols representing individual neurons (FA-1, blue; SA-1, red), and bars indicate mean values across all neurons.

$\mathrm{mm} / \mathrm{s}$, respectively. With increasing speed, the spikes were generated for shorter periods, yet the mean firing rate did not increase proportionate to speed because of the decreasing ratio of number of spikes per scan to speed. For the peak rate, the speed effect was modest. Averaged across all 16 neurons, the peak rate was $210 \pm 53,245 \pm 66$, and $244 \pm 53 \mathrm{~Hz}$ at 15, 30, and $60 \mathrm{~mm} / \mathrm{s}$, respectively.

Despite the fact that between the mappings at the different speeds, a neuron was subjected to 15 scans involving another pattern of raised elements causing generation of several thousands of action potentials (see Materials and Methods), a neuron's maps obtained at the different scanning speeds were strikingly similar. Averaged across all 16 neurons, the correlation $\left(R^{2}\right)$ was $0.83,0.76$, and 0.74 for speed combinations 15 and 30 , 30 and 60 , and 15 and $60 \mathrm{~mm} / \mathrm{s}$, respectively (Fig. 6B, "between speeds" correlation). Yet, the correlations were somewhat weaker than the correlations between the empirical maps for the speed within the speed-pair that showed the lowest correlation (Fig. $6 B$, compare "between speeds" and "within speed" correlations). To critically address whether a neuron's subfield layout was preserved across speeds, we investigated whether between-speed correlations within neurons were significantly higher than the mean of correlations obtained with $180^{\circ}$ rotation of corresponding maps. Strikingly, for each neuron and all speed combinations the between-speed correlation was distinctly higher (Fig. 6B, compare "between-speeds" and " $180^{\circ}$ rotation"). The betweenspeed correlation and the correlation involving $180^{\circ}$ map rotation was significantly different, which verified this speed invariant characteristics of the sensitivity topography $\left(F_{(1,14)}=103.0, p\right.$ $<0.0001)$. Neither speed combination nor neuron type showed a statistically significant effect on the difference $\left(F_{(2,28)}=3.1\right.$, $p=0.06$ and $F_{(1,14)}=0.41, p=0.53$, respectively) and there was no interaction effect between speed combination and neuron type $\left(F_{(2,28)}=0.02, p=0.98\right)$.

Next, we asked how well the distinctiveness of a neuron's receptive field properties across speeds is maintained with reference to other neurons' fields. For each neuron, we cross-correlated the map obtained at each speed with the neuron's own maps obtained at the other two speeds and with the maps obtained for all other neurons at each speed ( 3 speeds $\times 16$ neurons $-1=47$ correlations/speed). We then assessed for each speed how often the highest and the second highest correlation were found among the same neuron's maps obtained at another speed. Strikingly, the maps of all 16 neurons and at all three speeds were most similar to a map of the same neuron obtained at one of the other two speeds. Even for just one speed, the probability would be practically zero for this to happen by chance [ $p$ $\left.=(2 / 47)^{16}\right]$. Moreover, for 39 out of the 48 maps, the secondhighest correlation was also found with a map of the same neuron, again an outcome that by chance would be virtually zero. We did not find an effect of neuron type on the frequency of cases where the second-highest correlation was with a map of another neuron $\left(\chi_{1}^{2}=1.99, p=0.158\right)$.

Taken together, these results show that a neuron's receptive field sensitivity topography was largely invariant across tested scanning speeds and that the particularities of the receptive field properties relative to other neurons receptive fields essentially are maintained across speeds. This is in line with previous indications that the spatial structuring of FA-1 and SA-1 responses to scanned raised tactile elements is substantially maintained at speeds up to at least $90 \mathrm{~mm} / \mathrm{s}$ (Phillips et al., 1992; Pruszynski and Johansson, 2014).

\section{Conservation of receptive field sensitivity topography across} scanning directions

To examine the stability of the subfield layout across scanning directions, we compared maps generated with 0.1-mm kernel width for scans at $30 \mathrm{~mm} / \mathrm{s}$ in the proximal-distal and distal- 
proximal directions. Data from 22 neurons (16 FA-1s, 6 SA-1s) were analyzed, 11 of which (8 FA-1s, 3 SA-1s) were recorded in the present experiment and the remaining 11 (8 FA-1s, 3 SA-1s) in a previous series of experiments (Pruszynski and Johansson, 2014). For the neurons of the present experiments, for each direction the map used was an average of the maps obtained by the three scans, whereas for the remaining neurons only one map was available for each direction. For the neurons of the present experiment, the estimated subfield spatial acuity did not differ significantly between the two scanning directions $\left(t_{(10)}=\right.$ $0.68, p=0.51 ; t$ test for dependent samples).

On visual inspection of a neuron's maps for the two directions, apparently homologous subfields could usually be identified, but their relative positions in the receptive field could differ between the maps (Fig. 7A, top panels). That is, compared with one of the maps, the map of the opposite direction appeared to be subject to different degrees of compression, stretching and shear, and could even appear slightly rotated. Such warping would be consistent with the neurons having ridge-associated receptors and that direction-dependent shear deformations of the ridge pattern of varying complexity occur when a surface slides over the fingertip skin (Delhaye et al., 2016).

To quantitatively examine the consistency of the subfield layout across the scanning directions in the face of map warping, we performed an analysis where we sought to factor in some aspects of the warping. First, we thresholded the maps to $50 \%$ of the maximum value to focus on highly sensitive zones (Fig. $7 A a)$. We then transformed the map obtained in the distal-proximal scanning direction to best resemble that obtained in the proximal-distal direction as judged by cross-correlation (Fig. $7 A b)$. The parameters of the transformation involved stretching/ compressing the entire map both in the scanning direction and in its perpendicular direction and rotation of the map. By changing the values of these parameters with small steps and in different combinations, the coefficients that gave the best correlation were determined and used for the transformation (see Materials and Methods). Although this transformation did not offset shear deformations of the skin surface within the receptive fields, for each neuron type it generally resulted in visually fairly similar maps for the two directions (Fig. 7B). Moreover, the pairwise correlation between a neuron's maps of the two scanning direction was regularly higher than that between the map of the proximal-distal direction and the same map rotated $180^{\circ}\left(F_{(1,20)}=\right.$ 38.9, $p<0.0001$; Fig. $7 C)$. This indicated that neurons' subfield structure was largely preserved over scanning directions.

We finally considered how well a neuron's receptive field maintains its distinctive character over other neurons' fields across scanning directions. We cross-correlated each neuron's processed map with its map for the opposite scanning direction and with the corresponding maps obtained for all other neurons in both directions $(2 \times 43$ correlations $)$. We then evaluated how frequently among neurons the highest correlation existed for the same neuron's maps. Of all 22 neurons we found this happened for 17 and 18 neurons in the proximal-distal and distal-proximal direction, respectively. The chance, at the population level, for this outcome would be virtually zero if the neurons' distinctiveness regarding receptive field properties would have been lost with the change in scanning direction $(p<0.0001$; binominal test).

Taken together, these results suggest that the internal sensitivity topography of a neuron's receptive field was largely conserved across scanning directions but could be influenced by directiondependent shear deformations of the skin surface. In addition, most neurons retain the distinctiveness of the features of their receptive fields with reference to other neurons' fields.

\section{Discussion}

Our results indicate that the spatial sensitivity of the receptive field subfield arrangement of FA-1 and SA-1 neurons innervating human fingertips is in the submillimeter range. The subfield acuity as well as the subfield layout appear similar across the tested scanning speeds and the modest speed effect on maximum firing rate indicates that the spatial structuring of neurons' responses is well maintained even at low speeds. The estimated spatial acuity is also similar across scanning directions, but the subfields can be displaced relative to one another to some extent depending on direction. We interpret this observation as the subfields staying at fixed places on the skin surface while their relative displacement reflecting complex direction-dependent shear deformations of the skin surface and its ridge pattern (Delhaye et al., 2016).

The similar dimensions of the papillary ridges and the neurons' subfields and their estimated spatial acuity suggests that the ridge-associated receptor organ representing a subfield measures mechanical events at an individual ridge. Such spatial selectivity might be achieved by a combination of the structural compartmentalization of the ridged skin and the ridge-governed contact mechanics of the fingertip. As for the structure, the subfield receptor selectivity matching the width of a ridge could be explained by the limiting (adhesive) ridges anchoring the papillary ridges to deeper tissues (Cauna, 1954; Halata, 1975) allowing a ridge to be laterally deflected without appreciably affecting its neighbors (Johansson and LaMotte, 1983; LaMotte and Whitehouse, 1986; Lee et al., 2020). The transverse ridges protruding into the dermis and mechanically separating the dermal papillae from each other along a ridge (Cauna, 1954; Halata, 1975) might explain that the spatial selectivity of the receptor organs appeared similarly high when the stimulation dots moved along a ridge as in its transverse direction, i.e., the movement direction of the dots in relation to the orientation of the ridges barely influenced neurons' subfield acuity.

Concerning contact mechanics, the sliding of the stimulus surface meant that frictional forces acted on skin ridges, which usually applies during object manipulation and tactile exploratory tasks (Adams et al., 2013). For smooth parts of the stimulus surface, adhesive frictional forces were likely distributed similarly over microscopic contact zones at the peaks of individual ridges (Soneda and Nakano, 2010; Delhaye et al., 2016) whereas the moving dots likely caused local phasic distortions of consecutive ridges through interlocking, plowing, and hysteresis friction (Johansson and LaMotte, 1983; LaMotte and Whitehouse, 1986; Tomlinson et al., 2011; Derler and Gerhardt, 2012; Van Kuilenburg et al., 2013; Chimata and Schwartz, 2015; Lee et al., 2020). As such, skin deformations caused by irregularities in a sliding surface excite primate ridge-associated tactile neurons much more effectively than comparable stimuli perpendicularly indented into the skin (Vallbo and Hagbarth, 1968; Johnson and Lamb, 1981; Phillips et al., 1983; LaMotte and Whitehouse, 1986; Johansson and Westling, 1987). Moreover, the sensitivity topography of FA-1 and SA-1 receptive fields exhibits deeper spatial modulation with sliding stimuli than with punctate perpendicular skin indentations (cf. current results and Johansson, 1978). These sensitivity improvements likely contribute to the increase in perceived intensity and clarity of tactile surface details during sliding movements compared with when we statically contact the 
A

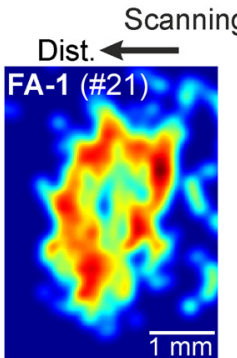

乃a

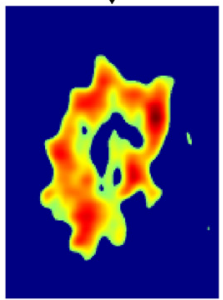

B
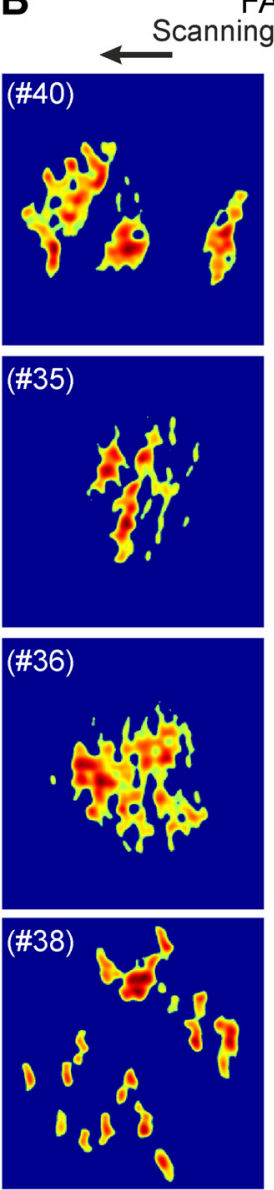

FA-1

ing direction
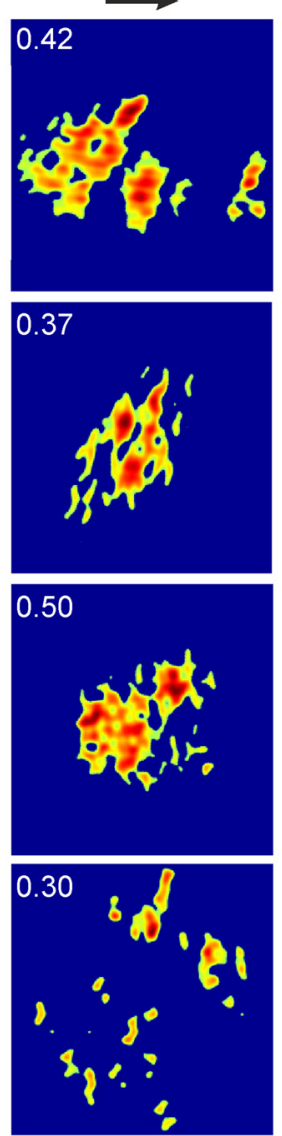

C

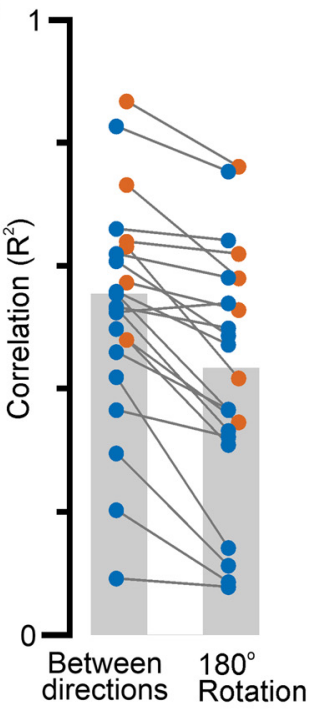

SA-1

Scanning direction
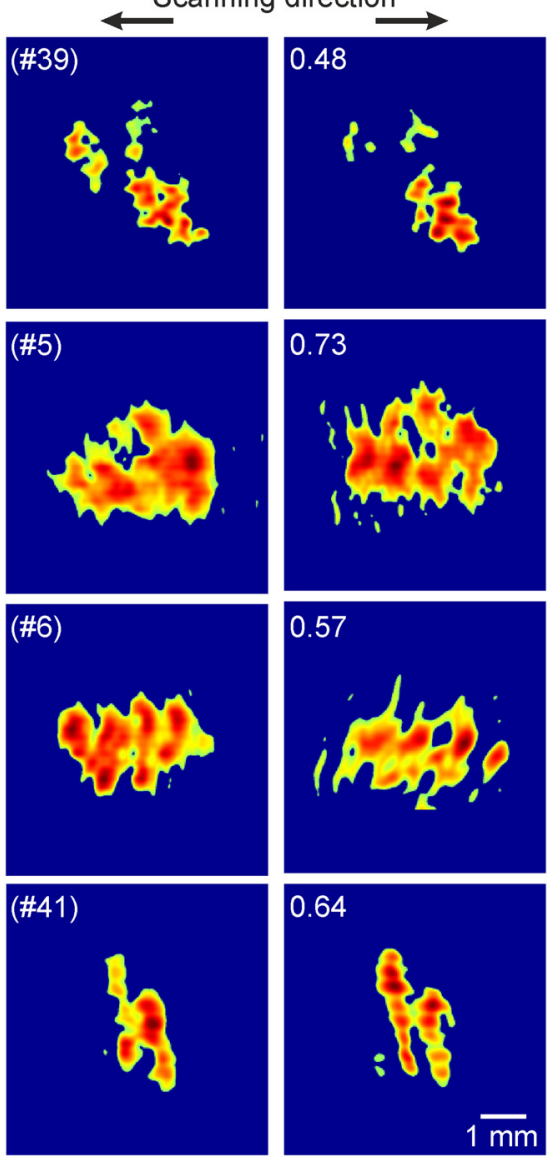

Figure 7. Effect of scanning direction on receptive field sensitivity topography examined at $30 \mathrm{~mm} / \mathrm{s}$ scanning speed. $A$, left and right top panels, Receptive field sensitivity topography of an exemplar FA-1 neuron obtained in proximal-distal and distal-proximal scanning direction, respectively. Left bottom panel, Proximal-distal map after thresholding (a) and right bottom panel the thresholded distal-proximal map that best matched the thresholded proximal-distal map after transformation ( $\boldsymbol{b}$; entire distal-proximal map was stretched in the scanning direction, compressed in its perpendicular direction and rotated counterclockwise). $\boldsymbol{B}$, Comparison of thresholded sensitivity maps of four exemplary neurons of each type obtained during proximal-distal and distal-proximal scanning after the latter had been transformed to best match the former. Numbers in the top left corners of the distal-proximal maps indicate, for each neuron, the correlation between the compared maps. C, Pairwise correlations within individual neurons between thresholded proximal-distal and distal-proximal maps after the latter had been transformed ("between directions") and between thresholded proximal-distal maps and the same maps rotated $180^{\circ}$ ("180 rotation"). Lines join symbols representing individual neurons (16 FA-1, blue; $6 \mathrm{SA}-1$, red) and bars indicate mean values across all neurons. 
same objects (Katz, 1925; Johansson and LaMotte, 1983; Lamb, 1983; Phillips et al., 1983; Loomis, 1985).

The current study has several limitations. These include methodological issues that may have resulted in an underestimation of the spatial acuity of neurons' subfields. First, mechanical changes in the fingertip with respiration and heartbeats (Johansson and Vallbo, 1979b) and varying creep of the skin during the repeated scans (drum revolutions) might have imposed noise in our SEPs by falsely increasing the spatial jitter of action potentials. Second, the similarity in dimension of the stimulation dots (top diameter $=0.4 \mathrm{~mm}$ ) and the estimated subfield acuity suggests that our probe could have acted as a spatial low-pass filter and thus contributed to an underestimation of the acuity. However, if the ridge deflections exciting receptor organs were primarily driven by the leading edge of the dots (LaMotte and Whitehouse, 1986), the size of the dot might have been of less importance for the estimated subfield acuity. Indeed, in previous experiments with scanned raised elements, we noted that both FA-1 and SA-1 neurons usually responded more intensely to the leading than to trailing ends of the elements (Pruszynski and Johansson, 2014), which is consistent with previous studies on analogous neurons in monkeys (Blake et al., 1997).

That the leading edge of the dots constituted the effective stimulation in tandem with the lateralized location of the Meissner bodies in dermal papillae on either side within the papillary ridges could explain that the width of the subfields for FA-1 neurons, and thus clustering of action potentials, sometimes appeared narrower than the ridge width (Figs. $1 E, 5 B$, neurons \#11, \#13, and \#14). That is, when dots pass over ridges, subfield receptors in papillae behind their ascending walls that primarily capture the dots should excite the neuron more intensely than receptors behind descending walls where stress and strain changes should be less intense. Consequently, depending on which side of a ridge a neuron's subfield receptor is located, the scanning direction could have affected the expression of a subfield, which may have contributed to the directional influence on neurons' subfield layout. The SA-1 neurons should show less similar directional effects since the Merkel complexes are centered relative to the papillary ridges (Cauna, 1954).

Methodological limitations prevented us from establishing direct links between deformation changes of individual ridges and nerve signals. These limitations concerned our video monitoring system (see Materials and Methods) but also that times for cutaneous mechanical stimulus transmission, receptor transduction and axonal spike conduction were not measured. These unknown times, dominated by the conduction time because of the significant distance from the fingertips to the recording electrode in our study $(\sim 0.5 \mathrm{~m})$, generated a positional shift in the scanning direction of SEP relative to the skin which increased with scanning speed. Given that the axonal conduction velocity varies between $\sim 25$ and $\sim 70 \mathrm{~m} / \mathrm{s}$ among FA-1 and SA-1 neurons (Mackel, 1988; Kakuda, 1992), depending on neuron, the conduction time could cause a SEP shift between $\sim 0.2$ and $\sim 0.6$ $\mathrm{mm}$ at $30 \mathrm{~mm} / \mathrm{s}$ scanning speed, i.e., for some neurons a shift of more than one ridge width.

Other limitations concern the generalizability of the results. The present and previous functional studies of the subfield arrangement of the FA-1 and SA-1 neurons are based on scanned stimuli limited to $\sim 0.5$-mm-high embossed elements with trapezoidal cross sections (Phillips et al., 1992; Pruszynski and Johansson, 2014). Thus, little is known about how this arrangement is expressed in responses of FA-1 and SA-1 neurons to scanned geometric stimuli with different sizes, curvatures, and sharpness etc. Although, effects of such parameters have been studied in analogous neurons of monkeys (usually referred to as RA and SA; LaMotte and Srinivasan, 1987a,b; LaMotte et al., 1994; Blake et al., 1997), the results cannot be translated to humans because their receptive fields rarely exhibit a corresponding heterogeneous internal sensitivity topography featuring multiple subfields (Johnson and Lamb, 1981; Phillips and Johnson, 1981a; LaMotte and Whitehouse, 1986; LaMotte et al., 1994; Blake et al., 1997; Suresh et al., 2016). Similarly, the utility of the subfield arrangement of FA-1 and SA-1 neurons for encoding fine texture during tactile exploration is unknown. For example, for the FA-1 neurons that are exceptionally sensitive to local skin distortions, the prevailing view derived from monkey studies is that they only signal temporal information about vibrations that propagate openly through the skin (Phillips and Johnson, 1985; Yoshioka et al., 2001; Weber et al., 2013; Lieber et al., 2017). Although patterns of distinct local mechanical interactions between texture elements and individual papillary ridges induce such vibrations (Prevost et al., 2009; Scheibert et al., 2009; Fagiani et al., 2011; Manfredi et al., 2014; Chimata and Schwartz, 2015), a possible contribution from a population code comprising spatially modulated patterns of nerve activity leveraged by the spatial selectivity of neurons' subfields has not been considered. The existence of such a spatial code might help to explain a still unsolved problem, namely how texture perception can be invariant over a wide range of scanning speeds (Katz, 1925; Weber et al., 2013; Boundy-Singer et al., 2017). However, a central yet unresolved issue in this context is how human FA-1 and SA-1 neurons combine signals from their subfields when a fingertip scans textured surfaces. Interactions of activity originating in separated tactile receptors innervated by a single myelinated axon have been studied mainly in hairy skin of animals and the results suggest several types of possible nonlinear interactions and that these might differ between neuron types (Lindblom and Tapper, 1966; Grigg, 1986; Looft, 1988; Goldfinger, 1990; Lesniak et al., 2014).

The inability of an individual neuron to signal which of its subfields are primarily stimulated does not preclude the possibility that a population of neurons can signal tactile stimuli at subfield resolution (Pruszynski and Johansson, 2014; Pruszynski et al., 2018; Hay and Pruszynski, 2019). The key is that subfields belonging to different neurons are highly intermingled and partially overlap because receptive fields of neurons heavily overlap (Johansson and Vallbo, 1980). Hence, when an object is touched, neurons whose subfields spatially coincide with salient tactile features are primarily excited, while in a slightly different spatial stimulus configuration, another subset of neurons, which can share members with the first subset, is primary excited. Theoretically, for the FA-1 population innervating the fingertips, where all dermal papillae contain Meissner bodies, the resolution of such a spatial coincidence code would approach the distance between adjacent dermal papillae as about half of them are innervated by axonal branches originating from more than one neuron (Matsuoka et al., 1983; Nolano et al., 2003). This view on neural encoding of geometric structures of object surfaces differs radically from that of the generally accepted model concerning human fingertips, which is based on neural data obtained from monkeys (Phillips and Johnson, 1981b; Van Boven and Johnson, 1994; Khalsa et al., 1998; Johnson et al., 2000; Goodwin and Wheat, 2004; Yau et al., 2016; Saal et al., 2017). First, by assuming that receptive fields of the relevant neurons have Gaussian- 
like sensitivity profiles with a single point of maximum sensitivity, this model does not recognize a potential contribution from multifocal receptive fields to fingertip spatial sensitivity. Spatial resolution at the neural population level relies on pixel-like isomorphic spatial representations of tactile features and is essentially limited by the estimated spacing between receptive field centers $(\sim 1 \mathrm{~mm})$. However, if respecting the multifocal nature of the receptive fields, the theoretical limit of spatial resolution at a given skin innervation density is defined by the much smaller distance between neurons' interdigitating subfields. Accordingly, the subfield arrangement may provide a straightforward explanation for a spatial resolution better than predicted by the sampling theorem (see Introduction) as opposed to the proposed complex spatial interpolation scheme based on the brain computing relative discharge rates of neurons with neighboring overlapping Gaussian-like receptive fields (Loomis and Collins, 1978; Wheat et al., 1995; Friedman et al., 2002). Second, by focusing on the SA-1 neurons as the essential contributor to the high spatial resolution of the fingertips, the generally accepted model largely ignores contributions from FA-1 neurons although they show a similarly high spatial sensitivity and, at the population level, could contribute more information than SA-1 neurons because of their much higher density in the fingertips (Johansson and Vallbo, 1979a).

However, sampling spatial tactile patterns with first-order neurons receiving converging inputs from multiple subfields cannot allow for complete reconstruction of any pattern with subfield resolution. Nevertheless, given the sparsity in biologically relevant signaling patterns, functional spatial resolution corresponding to the subfield acuity could be achieved by the brain for behaviorally relevant stimuli by mechanisms analogous to those already identified functioning in sensory systems generally (Olshausen and Field, 2004; Barranca et al., 2014; Yamins et al., 2014; Pruszynski et al., 2018; Rongala et al., 2018; Zhao et al., 2018).

\section{References}

Adams MJ, Johnson SA, Lefèvre P, Lévesque V, Hayward V, André T, Thonnard JL (2013) Finger pad friction and its role in grip and touch. J R Soc Interface 10:20120467.

Barranca VJ, Kovačič G, Zhou D, Cai D (2014) Sparsity and compressed coding in sensory systems. PLoS Comput Biol 10:e1003793.

Birznieks I, Jenmalm P, Goodwin AW, Johansson RS (2001) Encoding of direction of fingertip forces by human tactile afferents. J Neurosci 21:8222-8237.

Blake DT, Johnson KO, Hsiao SS (1997) Monkey cutaneous SAI and RA responses to raised and depressed scanned patterns: effects of width, height, orientation, and a raised surround. J Neurophysiol 78:2503-2517.

Boundy-Singer ZM, Saal HP, Bensmaia SJ (2017) Speed invariance of tactile texture perception. J Neurophysiol 118:2371-2377.

Brown AG, Iggo A (1967) A quantitative study of cutaneous receptors and afferent fibres in the cat and rabbit. J Physiol 193:707-733.

Cauna N (1954) Nature and functions of the papillary ridges of the digital skin. Anat Rec 119:449-468.

Cauna N (1959) The mode of termination of the sensory nerves and its significance. J Comp Neurol 113:169-209.

Chimata GP, Schwartz CJ (2015) Investigation of friction mechanisms in finger pad sliding against surfaces of varying roughness. Biotribology 3:1119.

Delhaye B, Barrea A, Edin BB, Lefèvre P, Thonnard JL (2016) Surface strain measurements of fingertip skin under shearing. J R Soc Interface 13:20150874.

Derler S, Gerhardt LC (2012) Tribology of skin: review and analysis of experimental results for the friction coefficient of human skin. Tribol Lett 45:1-27.
Dillon YK, Haynes J, Henneberg M (2001) The relationship of the number of Meissner's corpuscles to dermatoglyphic characters and finger size. J Anat 199:577-584.

Edin BB, Bäckström PA, Bäckström LO (1988) Single unit retrieval in microneurography: a microprocessor-based device controlled by an operator. J Neurosci Methods 24:137-144.

Fagiani R, Massi F, Chatelet E, Berthier Y, Akay A (2011) Tactile perception by friction induced vibrations. Tribol Int 44:1100-1110.

Fellous JM, Tiesinga PH, Thomas PJ, Sejnowski TJ (2004) Discovering spike patterns in neuronal responses. J Neurosci 24:2989-3001.

Friedman RM, Khalsa PS, Greenquist KW, LaMotte RH (2002) Neural coding of the location and direction of a moving object by a spatially distributed population of mechanoreceptors. J Neurosci 22:9556-9566.

Galton F (1892) Finger prints. London: Macmillan and Co.

Gamzu E, Ahissar E (2001) Importance of temporal cues for tactile spatialfrequency discrimination. J Neurosci 21:7416-7427.

Goldfinger MD (1990) Random-sequence stimulation of the G1 hair afferent unit. Somatosens Mot Res 7:19-45.

Goodwin AW, Wheat HE (2004) Sensory signals in neural populations underlying tactile perception and manipulation. Annu Rev Neurosci 27:53-77.

Grigg P (1986) Biophysical studies of mechanoreceptors. J Appl Physiol (1985) 60:1107-1115.

Halata Z (1975) The mechanoreceptors of the mammalian skin ultrastructure and morphological classification. Adv Anat Embryol Cell Biol 50:3-77.

Hay E, Pruszynski JA (2019) Orientation processing by synaptic integration across first-order tactile neurons. bioRxiv. doi: 10.1101/396705.

Hollins M, Bensmaïa SJ (2007) The coding of roughness. Can J Exp Psychol 61:184-195.

Johansson RS (1978) Tactile sensibility in the human hand: receptive field characteristics of mechanoreceptive units in the glabrous skin area. J Physiol 281:101-125.

Johansson RS, Vallbo AB (1979a) Tactile sensibility in the human hand: relative and absolute densities of four types of mechanoreceptive units in glabrous skin. J Physiol 286:283-300.

Johansson RS, Vallbo AB (1979b) Detection of tactile stimuli. Thresholds of afferent units related to psychophysical thresholds in the human hand. J Physiol 297:405-422.

Johansson RS, Vallbo AB (1980) Spatial properties of the population of mechanoreceptive units in the glabrous skin of the human hand. Brain Res 184:353-366.

Johansson RS, LaMotte RH (1983) Tactile detection thresholds for a single asperity on an otherwise smooth surface. Somatosens Res 1:21-31.

Johansson RS, Westling G (1987) Signals in tactile afferents from the fingers eliciting adaptive motor responses during precision grip. Exp Brain Res 66:141-154.

Johnson KO, Lamb GD (1981) Neural mechanisms of spatial tactile discrimination: neural patterns evoked by braille-like dot patterns in the monkey. J Physiol 310:117-144.

Johnson KO, Phillips JR (1981) Tactile spatial resolution. I. Two-point discrimination, gap detection, grating resolution, and letter recognition. J Neurophysiol 46:1177-1192.

Johnson KO, Yoshioka T, Vega-Bermudez F (2000) Tactile functions of mechanoreceptive afferents innervating the hand. J Clin Neurophysiol 17:539-558.

Kakuda N (1992) Conduction-velocity of low-threshold mechanoreceptive afferent-fibers in the glabrous and hairy skin of human hands measured with microneurography and spike triggered averaging. Neurosci Res 15:179-188.

Katz D (1925) Der Aufbau der Tastwelt. Z Psychol Physiol Sinn 11:1-270.

Khalsa PS, Friedman RM, Srinivasan MA, LaMotte RH (1998) Encoding of shape and orientation of objects indented into the monkey fingerpad by populations of slowly and rapidly adapting mechanoreceptors. J Neurophysiol 79:3238-3251.

Kuehn ED, Meltzer S, Abraira VE, Ho CY, Ginty DD (2019) Tiling and somatotopic alignment of mammalian low-threshold mechanoreceptors. Proc Natl Acad Sci USA 116:9168-9177.

Lamb GD (1983) Tactile discrimination of textured surfaces: psychophysical performance measurements in humans. J Physiol 338:551-565.

LaMotte RH, Whitehouse J (1986) Tactile detection of a dot on a smooth surface: peripheral neural events. J Neurophysiol 56:1109-1128. 
LaMotte RH, Srinivasan MA (1987a) Tactile discrimination of shape: responses of rapidly adapting mechanoreceptive afferents to a step stroked across the monkey fingerpad. J Neurosci 7:1672-1681.

LaMotte RH, Srinivasan MA (1987b) Tactile discrimination of shape: responses of slowly adapting mechanoreceptor afferents to a step stroked across the monkey fingerpad. J Neurosci 7:1655-1671.

LaMotte RH, Srinivasan MA, Lu C, Klusch-Petersen A (1994) Cutaneous neural codes for shape. Can J Physiol Pharmacol 72:498-505.

Lederman SJ (1974) Tactile roughness of grooved surfaces: the touching process and effects of macro- and microsurface structure. Percept Psychophys 16:385-395.

Lee ZS, Maiti R, Carré MJ, Lewis R (2020) Morphology of a human finger pad during sliding against a grooved plate: a pilot study. Biotribology 21:100114.

Lesniak DR, Marshall KL, Wellnitz SA, Jenkins BA, Baba Y, Rasband MN, Gerling GJ, Lumpkin EA (2014) Computation identifies structural features that govern neuronal firing properties in slowly adapting touch receptors. Elife 3:e01488.

Lieber JD, Xia X, Weber AI, Bensmaia SJ (2017) The neural code for tactile roughness in the somatosensory nerves. J Neurophysiol 118:3107-3117.

Lindblom Y, Tapper DN (1966) Integration of impulse activity in a peripheral sensory unit. Exp Neurol 15:63-69.

Looft FJ (1988) Interdome interactions in cutaneous type I receptors. IEEE Trans Biomed Eng 35:973-980.

Loomis JM (1979) An investigation of tactile hyperacuity. Sens Processes 3:289-302.

Loomis JM (1985) Tactile recognition of raised characters: a parametric study. Bull Psychon Soc 23:18-20.

Loomis JM, Collins CC (1978) Sensitivity to shifts of a point stimulus: an instance of tactile hyperacuity. Percept Psychophys 24:487-492.

Mackel R (1988) Conduction of neural impulses in human mechanoreceptive cutaneous afferents. J Physiol 401:597-615.

Manfredi LR, Saal HP, Brown KJ, Zielinski MC, Dammann JF 3rd, Polashock VS, Bensmaia SJ (2014) Natural scenes in tactile texture. J Neurophysiol 111:1792-1802.

Matsuoka S, Suzuki H, Morioka S, Ogawa Y, Kojima T (1983) Quantitative and qualitative studies of Meissner's corpuscles in human skin, with special reference to alterations caused by aging. J Dermatol 10:205-216.

Neubarth NL, Emanuel AJ, Liu Y, Springel MW, Handler A, Zhang Q, Lehnert BP, Guo C, Orefice LL, Abdelaziz A, DeLisle MM, Iskols M, Rhyins J, Kim SJ, Cattel SJ, Regehr W, Harvey CD, Drugowitsch J, Ginty DD (2020) Meissner corpuscles and their spatially intermingled afferents underlie gentle touch perception. Science 368:eabb2751.

Nolano M, Provitera V, Crisci C, Stancanelli A, Wendelschafer-Crabb G, Kennedy WR, Santoro L (2003) Quantification of myelinated endings and mechanoreceptors in human digital skin. Ann Neurol 54:197-205.

Olczak D, Sukumar V, Pruszynski JA (2018) Edge orientation perception during active touch. J Neurophysiol 120:2423-2429.

Olshausen BA, Field DJ (2004) Sparse coding of sensory inputs. Curr Opin Neurobiol 14:481-487.

Paré M, Smith AM, Rice FL (2002) Distribution and terminal arborizations of cutaneous mechanoreceptors in the glabrous finger pads of the monkey. J Comp Neurol 445:347-359.

Peters RM, Hackeman E, Goldreich D (2009) Diminutive digits discern delicate details: fingertip size and the sex difference in tactile spatial acuity. J Neurosci 29:15756-15761

Phillips JR, Johnson KO (1981a) Tactile spatial resolution. II. Neural representation of Bars, edges, and gratings in monkey primary afferents. J Neurophysiol 46:1192-1203.

Phillips JR, Johnson KO (1981b) Tactile spatial resolution. III. A continuum mechanics model of skin predicting mechanoreceptor responses to bars, edges, and gratings. J Neurophysiol 46:1204-1225.

Phillips JR, Johnson KO (1985) Neural mechanisms of scanned and stationary touch. J Acoust Soc Am 77:220-224.

Phillips JR, Johnson KO, Browne HM (1983) A comparison of visual and two modes of tactual letter resolution. Percept Psychophys 34:243-249.

Phillips JR, Johansson RS, Johnson KO (1992) Responses of human mechanoreceptive afferents to embossed dot arrays scanned across fingerpad skin. J Neurosci 12:827-839.
Prevost A, Scheibert J, Debrégeas G (2009) Effect of fingerprints orientation on skin vibrations during tactile exploration of textured surfaces. Commun Integr Biol 2:422-424.

Provitera V, Nolano M, Pagano A, Caporaso G, Stancanelli A, Santoro L (2007) Myelinated nerve endings in human skin. Muscle Nerve 35:767775 .

Pruszynski JA, Johansson RS (2014) Edge-orientation processing in firstorder tactile neurons. Nat Neurosci 17:1404-1409.

Pruszynski JA, Flanagan JR, Johansson RS (2018) Fast and accurate edge orientation processing during object manipulation. Elife 7:e31200.

Rongala UB, Spanne A, Mazzoni A, Bengtsson F, Oddo CM, Jörntell H (2018) Intracellular dynamics in cuneate nucleus neurons support selfstabilizing learning of generalizable tactile representations. Front Cell Neurosci 12:210.

Saal HP, Delhaye BP, Rayhaun BC, Bensmaia SJ (2017) Simulating tactile signals from the whole hand with millisecond precision. Proc Natl Acad Sci USA 114:E5693-E5702.

Scheibert J, Leurent S, Prevost A, Debregeas G (2009) The role of fingerprints in the coding of tactile information probed with a biomimetic sensor. Science 323:1503-1506.

Schreiber S, Fellous JM, Whitmer D, Tiesinga P, Sejnowski TJ (2003) A new correlation-based measure of spike timing reliability. Neurocomputing 52-54:925-931.

Smith AM, Gosselin G, Houde B (2002) Deployment of fingertip forces in tactile exploration. Exp Brain Res 147:209-218.

Soneda T, Nakano K (2010) Investigation of vibrotactile sensation of human fingerpads by observation of contact zones. Tribol Int 43:210-217.

Suresh AK, Saal HP, Bensmaia SJ (2016) Edge orientation signals in tactile afferents of macaques. J Neurophysiol 116:2647-2655.

Tomlinson SE, Carré MJ, Lewis R, Franklin SE (2011) Human finger contact with small, triangular ridged surfaces. Wear 271:2346-2353.

Vallbo ÅB, Hagbarth KE (1968) Activity from skin mechanoreceptors recorded percutaneously in awake human subjects. Exp Neurol 21:270289.

Vallbo ÅB, Johansson RS (1984) Properties of cutaneous mechanoreceptors in the human hand related to touch sensation. Hum Neurobiol 3:3-14.

Vallbo ÅB, Olausson H, Wessberg J, Kakuda N (1995) Receptive field characteristics of tactile units with myelinated afferents in hairy skin of human subjects. J Physiol 483:783-795.

Van Kuilenburg J, Masen MA, Van der Heide E (2013) The role of the skin microrelief in the contact behaviour of human skin: contact between the human finger and regular surface textures. Tribol Int 65:81-90.

Van Boven RW, Johnson KO (1994) The limit of tactile spatial-resolution in humans - grating orientation discrimination at the lip, tongue, and finger. Neurology 44:2361-2366.

Vázquez Y, Salinas E, Romo R (2013) Transformation of the neural code for tactile detection from thalamus to cortex. Proc Natl Acad Sci USA 110: E2635-E2644

Vega-Bermudez F, Johnson KO, Hsiao SS (1991) Human tactile pattern recognition: active versus passive touch, velocity effects, and patterns of confusion. J Neurophysiol 65:531-546.

Weber AI, Saal HP, Lieber JD, Cheng JW, Manfredi LR, Dammann JF, Bensmaia SJ (2013) Spatial and temporal codes mediate the tactile perception of natural textures. Proc Natl Acad Sci USA 110:17107-17112.

Wessberg J, Olausson H, Fernström KW, Vallbo AB (2003) Receptive field properties of unmyelinated tactile afferents in the human skin. J Neurophysiol 89:1567-1575.

Wheat HE, Goodwin AW, Browning AS (1995) Tactile resolution: peripheral neural mechanisms underlying the human capacity to determine positions of objects contacting the fingerpad. J Neurosci 15:5582-5595.

Yamins DL, Hong H, Cadieu CF, Solomon EA, Seibert D, DiCarlo JJ (2014) Performance optimized hierarchical models predict neural responses in higher visual cortex. Proc Natl Acad Sci USA 111:8619-8624.

Yau JM, Kim SS, Thakur PH, Bensmaia SJ (2016) Feeling form: the neural basis of haptic shape perception. J Neurophysiol 115:631-642.

Yoshioka T, Gibb B, Dorsch AK, Hsiao SS, Johnson KO (2001) Neural coding mechanisms underlying perceived roughness of finely textured surfaces. J Neurosci 21:6905-6916.

Zhao CW, Daley MJ, Pruszynski JA (2018) Neural network models of the tactile system develop first-order units with spatially complex receptive fields. PLoS One 13:e199196. 